\title{
Factores que dificultan titularse de una universidad mexicana
}

\section{Factors affecting students' graduation from a Mexican university}

\author{
Addy Rodríguez Betanzos \\ Doctora en Pedagogía. Docente en la Maestría en Educación en la Universidad de Quintana Roo, \\ México. Miembro del SIN.
}

Fecha de recibido: 17/02/2014

Fecha de aceptación: 11/06/2014

\section{Resumen}

Dos de cada diez jóvenes mexicanos tienen acceso a la educación terciaria y, sin embargo, una vez dentro, tres de cada diez estudiantes de educación terciaria no logran titularse. Y es que, si bien hay suficientes estudios sobre seguimientos de egresados, no es así sobre estudios de por qué los egresados no llegan a titularse. De ahí la relevancia de realizar un estudio descriptivo en una universidad pública y estatal a través de una encuesta a egresados de cinco generaciones de estudiantes que, habiendo finalizado sus planes de estudio no obtuvieron sus títulos, con el objetivo de identificar los factores que impiden, dificultan o retrasan la titulación.

PALABRAS CLAVE: Titulación - estudiantes universitarios - capital humano - investigación educativa - México - educación superior.

\section{Abstract}

Two out of ten Mexican students have access to a higher education institution and yet, once inside, three out of ten students fail to reach their bachelor's degree. Even if there are enough studies on graduates' follow-up, such is not the case on the issue why students do not graduate. Hence the relevance of a descriptive study, at a public State University and by means of a survey, of five generations of students which, having finished all their program courses would fail to reach their professional degrees. The aim of the present study is the identification of those factors that prevent, delay or hinder their degree acquisition.

KEYWORDS university degree - university students - human capital - educational research - Mexico - higher education

\section{Introducción}

A partir de mediados de la década de 1990, el sistema de educación superior mexicano se reestructuró de tal manera que en la actualidad las instituciones públicas de educación superior, particularmente todas las universidades públicas que se localizan en los 31 estados que conforman el territorio mexicano y que reciben el $50 \%$ o más de fondos federales que les otorga el gobierno nacional, cuentan con infraestructura moderna avalada por ISO 9000, modelos educativos actualizados y programas educativos acreditados por organismos de calidad, así como una planta docente certificada por el Programa de Mejora al Profesorado (PROMEP) y el Sistema Nacional de Investigadores del Consejo Nacional para la Ciencia y Tecnología (CONACyT). 
Sin embargo, existen aún graves problemas relacionados con la calidad educativa, entre ellos el déficit de eficiencia terminal, la falta de eficaces programas de seguimiento a los egresados universitarios y la no titulación de un significativo porcentaje de egresados que de una manera más o menos exitosa ya están insertos en el mercado laboral. Debido a ello, se ha investigado sobre la urgente necesidad de ofrecer educación universitaria de calidad. Asimismo, se han llevado a cabo estudios sobre la trayectoria educativa de los alumnos universitarios y el seguimiento de egresados. Empero son escasas las investigaciones sobre los factores que impiden la titulación de egresados universitarios. De ahí el interés por describir cuáles son los factores que impiden la titulación en una universidad pública localizada al sureste del territorio mexicano.

El artículo se divide en tres grandes apartados que se presentan a continuación: la presentación de la revisión de la literatura desde el marco teórico del capital humano; la descripción del estudio empírico realizado a cinco generaciones de alumnos que han egresado pero no se han titulado y, por último, los hallazgos de la encuesta realizada.

\section{Los universitarios son un activo de capital humano}

Edel, Duarte y Hernández (2005) señalan que los indicadores de la calidad en educación superior determinan que la eficiencia terminal representa una de las vías para alcanzar la productividad y los objetivos sociales. Aunque de forma contrastante, los procesos y lineamientos institucionales de las universidades se transforman, lo que les dificulta obtener un logro significativo en términos de titulación. Contar con un título universitario es imprescindible, no sólo para el acceso a mejores empleos, mejor nivel salarial y mayor status profesional, sino por el nivel de desarrollo económico y cultural de un país.

De acuerdo con Lugo (2003), para mediados de la segunda década del presente siglo la educación superior se verá obligada a superar la formación tradicional para el empleo con una formación a corto plazo donde se realice el trabajo en formas distintas, ante lo cual las instituciones de educación superior se tendrán que replantear esquemas de innovación curricular y una oferta de profesiones acorde a las tendencias de la sociedad y a las demandas del mercado laboral.

Valenti (2006) afirma que la educación superior per cápita se encuentra altamente correlacionada con el ingreso per cápita de un país, por lo que las universidades se hallan obligadas a asegurar la eficiencia terminal, dar el debido seguimiento a sus egresados y propiciar la titulación de los mismos. Desde la perspectiva del capital humano, las profesiones son una manera racional de organizar la sociedad y las actividades económico-laborales que realizan las personas.

En teoría, los universitarios titulados son personas que cuentan con mejores oportunidades para incursionar de manera más estable en el campo laboral. Norting (2007) señala que el concepto de capital humano es un término que se introdujo como un factor de producción para reflejar no sólo la cantidad sino también la calidad en el grado de formación y productividad de las personas involucradas en un proceso productivo. Por tal razón, una gran parte del crecimiento económico de las sociedades -a nivel mundial- se relaciona con el término en cuestión y se vincula al nivel educativo y de formación de los agentes económicos.

La teoría del capital humano sostiene que la persona con estudios universitarios obtendrá mayores beneficios a futuro. De cierta forma, desde esta perspectiva, hay que analizar la importancia que tiene el título universitario en la inmersión del campo laboral del egresado, ya que sin dicha cédula profesional no podría aspirar con total seguridad a mejores oportunidades de vida tanto económica como profesionalmente. Por ende, es evidente la importancia que tiene que una persona invierta en una educación formal ya que ésta le permitirá adquirir conocimientos específicos en la carrera seleccionada, así como habilidades y competencias para la toma de decisiones en su actividad laboral. 
Becker (1983) sostiene que la educación formal es no sólo aprender nuevos conocimientos sino también aprender a ser para que, a futuro y a lo largo del desarrollo de cada persona, ésta obtenga habilidades y capacidades necesarias para desenvolverse mejor como ser humano. Asimismo identificó que el conocimiento se crea en las empresas, laboratorios y universidades, pero se difunde por medio de las familias para ser finalmente utilizado en la producción de bienes y servicios, los cuales permiten el crecimiento poblacional de las naciones e impulsan el aumento en el campo laboral para quienes requieren de recursos humanos capacitados para trabajar en sus empresas.

De acuerdo a la teoría credencialista expuesta desde 1970 por Randall Colin, el egresado titulado tiene también una ventaja: las credenciales o certificados educativos (títulos universitarios) cumplen una función de selección, clasificación y asignación de los trabajadores en el mercado laboral, aunque se contrapone a los mecanismos específicos a través de los cuales la educación cumple esta función.

Como bien señala Rodríguez (2006), en algunos países que han tenido éxito en materia económica los modelos educativos están vinculados con la economía y el progreso socioeconómico, lo cual significa que al trazar políticas de educación intervienen los intereses de los diferentes sectores de la sociedad para construir un ambiente propicio para el desarrollo de los objetivos de cada país. Si no, mírese el proyecto de la UNESCO de ciudades digitales.

Si lo anterior no convence aún de la importancia de tener una profesión en el mercado laboral, la teoría de enfoque social mejor conocida como Teoría de la Cola acuñada por Piore (1983) corrobora que el título profesional incide en la decisión del mercado laboral a favor de los que han recibido mayor educación, mientras que los que poseen menor formación deben continuar haciendo cola por otra oportunidad de empleo. Esta teoría recalca la importancia de contar con un título universitario que acredite y apoye al egresado en el campo laboral, a fin de tener oportunidad de demostrar sus capacidades y habilidades obtenidas durante la realización de su educación formal y la experiencia que irá obteniendo en el transcurso de su desenvolvimiento dentro del trabajo.

Tal como lo señalan López, Salvo y García (1989), un título universitario define la profesión que las personas van a realizar, conlleva las funciones de su identidad profesional, así como las tareas más habituales en las que se plasman esas funciones. En cierto sentido, el universitario titulado tiene en mano una credencial que podrá presentar en el campo laboral a fin de hacer uso de los conocimientos adquiridos a lo largo de su formación universitaria.

Es de entender la importancia que tiene para toda universidad que sus alumnos egresen, en tiempo y forma, con su título profesional correspondiente. La universidad como institución certificada que brinda servicios educativos de calidad debe contar con programas educativos acreditados y mantener un adecuado índice de eficiencia terminal. Sólo así rinde cuentas claras a la sociedad puesto que cabe esperar que los titulados universitarios constituyan un colectivo privilegiado dada su capacitación especializada orientada a un ámbito específico de la actividad profesional.

La calidad educativa universitaria se evalúa no sólo con los servicios que ofrecen las universidades sino también en el logro de la eficiencia de sus servicios, la forma flexible de las opciones de aprendizaje y diversificación de éstas, sin olvidar la transformación de la manera de aprender y enseñar y, por último, el brindar buenos servicios a los alumnos y a la sociedad. A su vez, Frame (2004), en su estudio en ochenta y cuatro instituciones universitarias públicas y privadas mexicanas que ofrecen carreras con licenciatura y cuentan con programas educativos de más de cuatro años de duración concluye que la eficiencia terminal de las universidades públicas sigue siendo menor que el de las universidades privadas.

Por consiguiente, y como resultado de un estudio en 1988 y uno más en 2001 por parte de la Asociación Mexicana de Universidades e Instituciones de Educación Superior (ANUIES), las instituciones universitarias públicas del país consideraron fundamental contar con un área encargada del seguimiento de sus egresados, no únicamente para estar en constante contacto 
con ellos y ofrecerles educación continua, sino realizar estudios de factibilidad profesional y pertinencia laboral así como estudios de seguimiento en trayectos educativos como el de la eficiencia terminal'.

Anzaldo, Morales y Nolasco (2001), al indagar sobre el comportamiento de la eficiencia terminal en los programas de licenciatura en su universidad y su relación con las características de la vida académica, explican las causas que intervienen en el éxito o en el fracaso escolar, el abandono de los estudios y las condiciones que prolongan el tiempo establecido para concluir satisfactoriamente los mismos. En lo académico enfatizan los estilos de ser docente, cuando el profesor no encuentra cómo lograr articular su manera de ser docente con la ejecución de los planes de estudio, hecho que incide en el desconocimiento que el estudiante tiene de las metas y objetivos de la institución y de la carrera. Así también se detectó que la deserción escolar como problemática educativa no sólo afecta la movilidad y expectativas educacionales y laborales de los individuos desertores sino que también influye significativamente en las metas y objetivos trazados por la institución escolar.

Por su parte, Edel, Duarte y Hernández (2006) al analizar los factores institucionales y no institucionales que afectan la eficiencia terminal, señalan que la misma está determinada por cinco factores, a saber: el laboral, el diseño y planeación curricular, la vinculación institucional, la selección de los aspirantes y el factor familiar, los cuales afectaron la relación entre los índices de egreso y de titulación. Los dos factores más importantes fueron en referencia a lo laboral. Se refuerza la idea de que la obtención de un título universitario es una vía importante para el acceso al campo laboral, seguido del factor de vinculación institucional el cual representa para el universitario la oportunidad de poder relacionar la teoría con la práctica y, para la universidad, la identificación institucional de las necesidades de formación en su oferta con respecto a los sectores productivos y sociales. Con base en los resultados obtenidos, Edel, Duarte y Hernández presentan cuatro dimensiones de atención prioritarias que son, en orden de importancia: sistematizar la información de la trayectoria escolar de los alumnos, generar un proceso formal de seguimiento a egresados, determinar la estrategia institucional con el propósito de incrementar sus índices de titulación y, por último, establecer el estudio de la eficiencia terminal como un eje permanente de investigación institucional.

En lo referente a la titulación, con el propósito de identificar las causas de los bajos índices de titulación, López, Salvo y García (1989) encuentran que la rigidez en los mecanismos de acreditación, tanto académicos como administrativos, es la causa principal de los bajos índices de titulación. Los autores señalan que existe una tendencia generalizada a unificar los requisitos académicos y administrativos para la obtención del título, lo cual es una tendencia más política que académica en la valoración de las cifras estadísticas. Esto se debe a que es frecuente medir la calidad académica a partir de los índices de titulación y la eficiencia terminal, mientras los alumnos presentan carencias importantes en su formación académica tanto en lo referente a los aspectos teórico-metodológicos, como a la dificultad para integrar los conocimientos y transformar la realidad.

En resumen, la eficiencia terminal y el índice de titulación en la educación superior permiten observar los niveles de deficiencia que tienen las universidades, con el fin de buscar estrategias de cambio para lograr la calidad en la educación.

\section{Estudio empírico de la no titulación universitaria}

El término "Factor" es de origen latino y proviene de las matemáticas, pero en la presente investigación y en el ámbito educativo se define como un agente que propicia una causa y su consabido efecto. Factor es un elemento personal o institucional que interviene o no en determinada situación. Con relación a la investigación que se reporta en este artículo, el objetivo es identificar, a manera de estudio descriptivo, los factores que impiden la titulación por parte de los egresados universitarios de cinco generaciones seguidas. 
En las dos últimas décadas se han implementado en las universidades públicas mexicanas diversos procedimientos de titulación que se supone facilitan a los estudiantes el cumplir, en tiempo y forma, con su carga académica según los requerimientos. Se sostiene que el alumno que ingresa sigue la trayectoria académica que curricularmente ha sido trazada para él en la profesión de su elección y que se titulará en tiempo y forma ya que la propia institución ha creado los espacios y mecanismos académicos y administrativos para facilitarle su permanencia y egreso de la vida universitaria, incluso su inclusión a la vida laboral y desarrollo profesional. Las tutorías, el servicio social, las prácticas profesionales, los programas educativos acreditados, la habilitación del profesorado universitario y las becas así como la diversidad de opciones para la titulación que ofrece la institución universitaria asumen que el índice de eficiencia terminal será alto y que una gran cantidad de alumnos universitarios logrará la titulación.

Se piensa que el gusto por el conocimiento y la libertad de elegir una profesión propician que el alumno universitario obtenga un título profesional, por lo que su profesión permitirá al alumno enfrentarse a la vida laboral con una carrera acorde a su capacidad académica, incluso teniendo en cuenta que en la actualidad existe una gran sobrepoblación de profesionales en algunas áreas disciplinares, debido a la restricción que presenta el mercado laboral. Sin embargo, y pese a que desde su ingreso a la universidad los alumnos universitarios conocen y siguen su plan de estudios, llevan un programa de introducción a la universidad y cuentan con el apoyo de tutorías y otras áreas académico-administrativas que los apoyan -entre ellas un área de psicología-, las estadísticas institucionales indican un alto déficit de eficiencia terminal en muchas universidades públicas mexicanas. Por ello, no sólo hay un preocupante índice de deserción anual por razones no formalmente conocidas, sino que, al graduarse una generación específica, muchos egresados no se titulan por lo cual les es difícil lograr cerrar un ciclo para iniciar otra etapa de su vida.

En la actualidad, la educación superior es una necesidad para la formación educativa de todo ser humano, de ahí la idea básica de considerar a la educación como una inversión que realiza el individuo con el fin de incrementar su eficiencia productiva, sus ingresos económicos y su calidad de vida en general. Ser egresado titulado de nivel superior es en esta era de globalización una necesidad fundamental para enfrentar la competencia laboral. Según Romo (2003), es la síntesis de una etapa que se cierra y, sobre todo, la oportunidad de abrirse a nuevas perspectivas, es decir, no debe ser visto como un trabajo terminal sino como uno que abre perspectivas o cierra etapas para abrir nuevas.

Las estadísticas muestran que los alumnos que egresan de una universidad pública de tamaño pequeño (como la del presente estudio, que no cuenta en su totalidad con más de diez mil alumnos), mantienen un bajo índice de titulación pese a todos los esfuerzos institucionales de su Sistema Institucional de Gestión de la Calidad que les ofrece, por ejemplo, seminarios de titulación o exámenes generales.

La importancia de elevar los índices de titulación se debe no solamente al hecho de que una baja eficiencia terminal podría afectar la credibilidad como institución certificada, sino que pone un freno al propio desarrollo individual de los universitarios puesto que no les permite continuar con su formación académica ni aspirar a mejores puestos laborales. De ahí la importancia de conocer, por parte de los propios egresados universitarios, las causas de tan reducido número de egresados titulados.

Por consiguiente, se planteó: identificar si la necesidad de contar con un trabajo inmediato al término de los estudios es un factor que influye en el bajo índice de titulación de los egresados de dicha institución; determinar si el hecho de que la institución no ofrezca a los estudiantes dentro de sus planes de estudios asignaturas que los apoyen en la realización de su trabajo de tesis a lo largo de su carrera profesional es otro factor que influye en el bajo índice de titulación de sus estudiantes, y, finalmente; identificar si el no cumplir con el porcentaje de inglés obligatorio al término de sus estudios es un factor adicional que influye en el bajo índice de titulación de los alumnos universitarios. 
En relación al método, se realizó una investigación cuantitativa a través de una encuesta para conocer los factores que, según los egresados de cinco generaciones universitarias, impiden, dificultan o retrasan su titulación. La encuesta se diseñó en cuatro partes:

- En la primera se identificaba el sexo y el estado civil de los egresados.

- La segunda se dividió en dos áreas: la primera sobre los aspectos familiares de los egresados (el nivel de estudio, ocupación y nivel de ingreso de sus padres), y la segunda sobre los aspectos profesionales de los propios egresados donde resultaba importante identificar su trayectoria laboral.

- La tercera parte contenía la opinión de los egresados sobre su campo formativo. Asimismo contó con cuatro preguntas sobre la satisfacción respecto a sus estudios y la imagen de su universidad tanto en lo que se refiere a la calidad en la educación como en lo relativo a las posibilidades de empleo para sus egresados.

- La cuarta y última parte contenía varios aspectos específicos sobre la titulación, a saber: conocimientos, nivel de acuerdo, propuestas sobre las modalidades de titulación, dificultades para la titulación, si realizó su servicio social, si cumplió con su nivel obligatorio de inglés y la razón para no titularse. La encuesta se diseñó en cuatro partes y contó con cuarenta y siete ítems.

Tras la fase piloto de la encuesta -mediante la cual se obtuvo un Alfa de Cronbach de .814, a un grupo de treinta egresados que participaban en un seminario de titulación-, la encuesta fue avalada con base en el juicio de cinco expertos.

De una población total de mil ciento veintiún egresados no titulados pertenecientes a las cinco últimas generaciones de la institución universitaria, debido a la dificultad en localizar a egresados se optó por una muestra por conveniencia a ciento cincuenta egresados localizados aunque sólo se lograron recuperar ciento dos encuestas.

\section{Factores en voces de los propios egresados universitarios}

En el aspecto contextual, la edad de los ciento dos egresados comprendió a setenta y dos universitarios no mayores de treinta años, mientras que treinta de ellos no superan los cuarenta años; setenta y cuatro egresados manifestaron ser solteros y el resto casados. Si bien la mayoría de los egresados manifestaron que mientras estudiaban eran económicamente dependientes de sus propios padres o de alguna beca institucional, cuarenta egresados dijeron trabajar mientras estudiaban. De ellos, veinte indicaron haber tenido trabajos eventuales mientras estudiaban y el resto fueron alumnos de tiempo completo.

Dichos padres -indicaron los egresados- eran trabajadores de algún sector gubernamental, lo cual coincide con la mayoría de los propios egresados: setenta y siete egresados laboran también en el sector público. Con referencia al campo profesional de los propios encuestados, cincuenta y tres egresados indicaron que si bien trabajaban, su empleo no se relacionaba con los estudios universitarios llevados a cabo.

En lo que respecta al grado de satisfacción manifestada por los egresados encuestados, el sesenta por ciento de ellos indicó sentirse muy satisfecho con la formación universitaria proporcionada por la universidad: sólo un cuarenta por ciento manifestó sentirse insatisfecho. En cuanto a la imagen universitaria, setenta y dos de los egresados la calificaron como buena.

Lo anterior confirma lo que las estadísticas señalan sobre la posición que ocupa esta institución universitaria entre otras universidades públicas, estatales y autónomas. La institución universitaria está reconocida por todos sus programas educativos acreditados, su profesorado habilitado con el perfil PROMEP (casi el sesenta por ciento de los mismos pertenece al Sistema Nacional de Investigadores del CONACYT), y los departamentos académicos siguen un proceso riguroso de selección de los estudiantes de nuevo ingreso a la universidad y se apoyan en el 
Programa Institucional de Tutorías donde los tutores dan puntual seguimiento a las trayectorias académicas individuales de sus alumnos. También se explica que la propia universidad sea parte de la poca oferta de educación superior que se proporciona a los jóvenes de una ciudad capital, dedicada la mayoría de su población a depender económicamente del sector público y de servicios, y que tanto los padres como los egresados laboren en dichos sectores.

En lo que respecta a la tercera parte de la encuesta, a través de veinticuatro preguntas se solicita la opinión de los egresados sobre su campo formativo, la satisfacción respecto a sus estudios y la imagen de su universidad en lo que se refiere a la calidad en la educación y a las posibilidades de empleo para sus egresados. La información obtenida se muestra en cinco apartados.

Cuando se les preguntó en qué medida su formación los preparó para laborar en distintos sectores de la economía, para asegurar un trabajo acorde, para generar propuestas de inversión o vincularse con el mercado laboral, y para trabajar de forma independiente, con base en la media estadística obtenida de cuatro por ciento los egresados indicaron que la profesión que eligieron les ayuda para encontrar un trabajo acorde a la misma, en diversas áreas del mercado laboral e incluso para trabajar por cuenta propia.

La opinión de los egresados con respecto al énfasis que pone su plan de estudios en alguno de estos elementos -teóricos, práctico-instrumentales, metodológicos de investigación, técnicos o de campo- indica que la media estadística más alta obtenida es un plan de estudios que enfatiza la parte práctico-instrumental, seguida por el trabajo de campo y los aspectos metodológicos o de investigación. No así los elementos teóricos ni técnicos, aspectos que habrá de reforzar en un futuro inmediato.

Para conocer en qué medida el plan de estudios les había proporcionado conocimientos, habilidades, capacidades, recursos para resolución de problemas y actitudes emprendedoras, se obtuvo la media más alta con casi cinco por ciento en lo que respecta a las actitudes emprendedoras, mientras que la mínima fue casi del cuatro por ciento en lo referente a las capacidades para la identificación y resolución de problemas. Según esto, y vinculado al aspecto anterior, los egresados indican que sus planes de estudios no les proporcionan los suficientes elementos teóricos ni enfatizan la capacidad de resolver los problemas o dificultades que se les presentan en el trabajo. Sin embargo, el que no se hayan titulado demuestra que personalmente carecen de esta capacidad.

En suma, como calificaba cada apartado curricular de su plan de estudios, en referencia a los estudios generales, divisionales, de concentración profesional de apoyo e idiomas, los egresados indicaron lo siguiente: califican alto los idiomas, seguido de las asignaturas de tronco común y, en un mismo estadístico, las demás áreas de su plan de estudios. Esto refuerza lo anterior: a los planes de estudios les falta dedicar mayor vinculación de las asignaturas de concentración profesional con las necesidades del sector productivo.

Por último, en relación a tres últimos aspectos sobre la satisfacción e imagen de la universidad, setenta y un egresados mencionaron estar de muy a totalmente satisfechos de haber estudiado en la universidad; sólo treinta y un estudiantes mencionaron estar insatisfechos aunque coincide con que son los egresados cuyo trabajo actual no corresponde a los estudios universitarios realizados.

En cuanto a la imagen de su universidad en relación a la calidad educativa, ochenta y cinco de los encuestados señalaron que es bueno si no excelente; sólo once mencionaron regular y el resto -tres egresados- dijeron que era malo. En cuanto a la posibilidad de empleo que ofrece la universidad para sus egresados, muy pocos dijeron excelente, la mayoría de las respuestas por parte de los egresados señalaron regular con un cuarenta y tres por ciento de las respuestas, el treinta y cinco por ciento lo considera bueno y un dieciocho por ciento de malo a pésimo. Sin embargo, la tabla indica la frecuencia y porcentaje obtenidos con respecto a la imagen que tiene el egresado de su universidad en cuanto a la posibilidad de empleo. 


\begin{tabular}{|l|c|c|}
\hline \multicolumn{2}{|c|}{$\begin{array}{c}\text { Tabla 1. ¿Cómo percibes la imagen de tu universidad en lo que se refiere a las } \\
\text { posibilidades de empleo para sus egresados? }\end{array}$} \\
\hline \multicolumn{1}{|c|}{ Opción } & Frecuencia & $\%$ \\
\hline Excelente & 3 & 2.9 \\
\hline Bueno & 36 & 35.3 \\
\hline Regular & 44 & 43.1 \\
\hline Malo & 17 & 16.7 \\
\hline Pésimo & 1 & 1 \\
\hline Sin respuesta & 1 & 1 \\
\hline
\end{tabular}

Fuente: elaboración propia.

En relación a los nueve aspectos específicos sobre la titulación, es decir, las opiniones sobre los conocimientos, nivel de acuerdo y propuestas sobre las modalidades de titulación así como a las dificultades que los egresados identificaron para no titularse cabe mencionar los hallazgos empíricos. A la pregunta de si conocían o no las modalidades de titulación que su plan de estudios ponía a su elección, los egresados dijeron que sí las conocían (ochenta y cinco por ciento), que sí estaban de acuerdo (veinte por ciento) y que no tenían propuesta alguna; por lo que cuando se les preguntó sobre cuál de las cuatro modalidades más comunes les parecía mejor lo indicaron con el siguiente orden de frecuencia: falta de tiempo, falta de asesores calificados, falta de bibliografía y problemas personales, problemas económicos. Sólo un egresado mencionó la falta de espacio.

\begin{tabular}{|l|c|c|}
\hline \multicolumn{2}{|c|}{ Tabla 2. Dificultades presentadas para No titularse } \\
\hline \multicolumn{1}{|c|}{ Opciones } & Frecuencia & $\%$ \\
\hline Falta de tiempo & 46 & 45.1 \\
\hline Falta de asesores calificados & 19 & 18.6 \\
\hline Falta de bibliografía & 14 & 13.7 \\
\hline Problemas personales & 14 & 13.7 \\
\hline Problemas económicos & 9 & 8.9 \\
\hline Otros & 4 & 3.9 \\
\hline Falta de espacio para trabajar & 1 & 1.0 \\
\hline
\end{tabular}

Fuente: elaboración propia.

Con referencia a si prestaron su servicio social y cumplieron con el requisito obligatorio del nivel de inglés indicaron no haberlo cumplido. Por último, al pedírseles especificasen la razón principal para no titularse -entre la falta de su propia organización, de la motivación necesaria del mismo profesorado hacia ellos en el proceso de titulación o todo en conjunto- cuarenta y cinco por ciento de los egresados señaló que los estudiantes no se titulan por falta de organización personal. 


\section{Gráfica 1. Razones de no titulación}

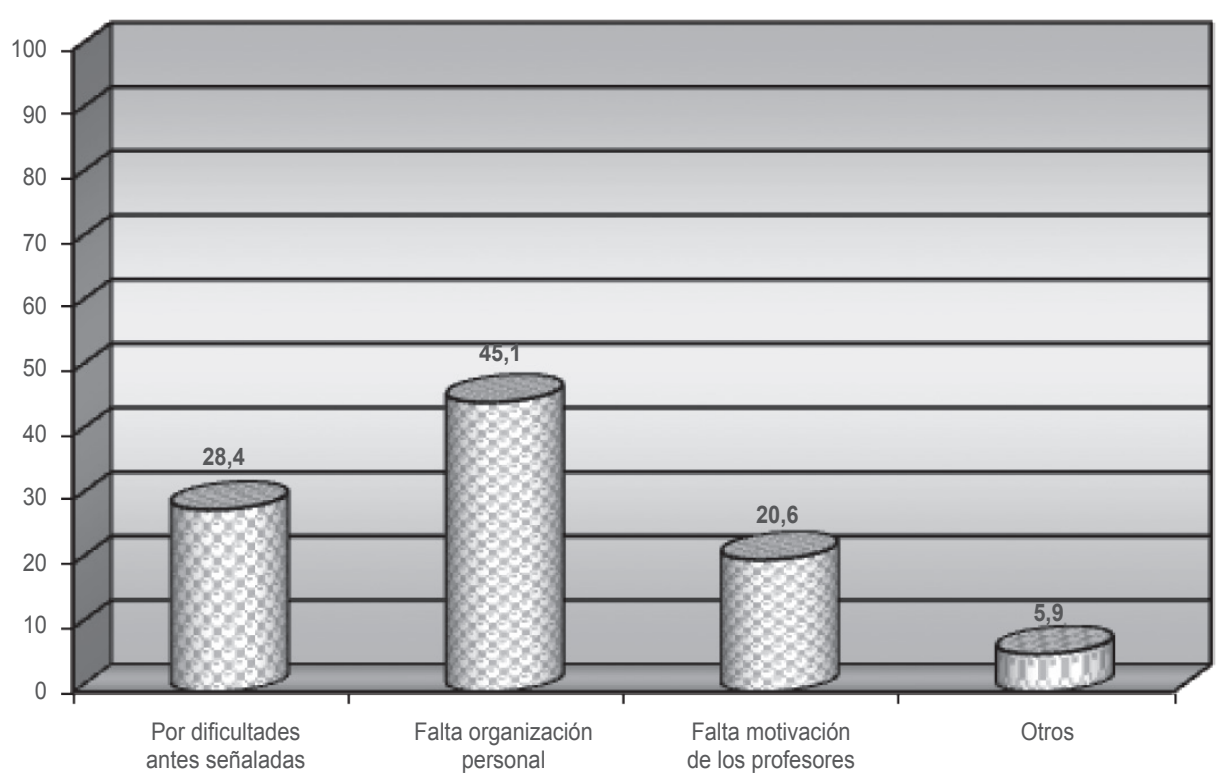

Fuente: elaboración propia.

Cotejando los resultados obtenidos a partir de la encuesta, se determinó que el inglés es el mayor problema que afecta a los estudiantes universitarios, pues al término de su programa de estudios tienen la obligación de haber cumplido con el nivel mínimo de inglés básico para poder culminar su carrera.

Los comentarios que figuran a continuación señalan de manera coloquial más que académicamente aceptable las inquietudes personales y sugerencias que podrían enriquecer los datos numéricos antes presentados.

- Obligarnos a comenzar la tesis dos semestres antes, ya que cuando uno termina la carrera sólo piensa en buscar empleo y no en la titulación.

- Incluir de manera obligatoria la vinculación al sector productivo.

- Instar a los universitarios a generar empresas.

- Realizar actividades para que egresados estén en contacto con egresados de la universidad. - Contratación de profesores más entregados a su profesión, con ética y responsabilidades.

- Más coordinación y orientación hacia los alumnos y sobre todo mucha comunicación para con ellos y buen trato.

Por esa razón se ha dejado sin más observaciones al respecto para pasar a las conclusiones de este proceso de indagación.

\section{Conclusiones}

Para responder al interrogante guía del presente estudio -indagación realizada mediante encuesta- se puede explicar que, debido a la importancia de contar con un título profesional que avale los estudios universitarios seguidos para desempeñarse en el mercado laboral la institución universitaria debe asegurarse de que los alumnos durante su trayectoria académica conozcan su plan de estudios, en particular los requisitos de titulación y las modalidades que la institución provee para facilitarles su egreso. Las tutorías se tornan cada vez más importantes en este sentido ya que las mismas constituyen la forma ideal de apoyar al alumno 
en la organización de su tiempo, en la elección de los asesores especializados y en el acceso y uso de recursos universitarios tales como la biblioteca, con el fin de elaborar una tesis o monografía, si ese fuera el caso.

Una vez que los alumnos egresan sin haber cumplido con todos los requisitos de su titulación y deciden incorporarse al mercado laboral sin haber obtenido el título profesional correspondiente tienen menos expectativas, interés o posibilidades de concluir un tramo de su vida profesional, lo que a la larga les ocasiona descontento, baja autoestima profesional o inestabilidad e incluso la posibilidad de perder mejores oportunidades laborales. De ahí que se propicie un círculo vicioso por parte del mismo egresado ya que, al no concluir sus estudios con el proceso de titulación, sus posibilidades de conseguir un trabajo acorde con sus estudios realizados son menores, así que terminará sintiéndose frustrado o complicándose más su vida futura. Para consideración del trabajo que realizan las autoridades universitarias, debe hacerse hincapié en la evaluación de los programas educativos, en las dificultades que implica realizar cada una de las modalidades de titulación -en particular una tesis (tiempo, dedicación, asesores y recursos, entre otros)- y considerar si sigue siendo vigente que ésta se realice como parte del proceso de titulación. Tal vez sea conveniente estructurar cursos de habilidades del pensamiento a través de los cuales, y mediante un proceso de indagación, puede llevarse a cabo una investigación transversal al mapa curricular incluyéndola en toda la trayectoria educativa y no únicamente al final.

Los estudios sobre eficiencia terminal, seguimiento de egresados y factores que influyen para la no titulación, más allá de enfatizar la importancia para un alumno universitario de contar con un título que le proporcione cierta certeza de insertarse mejor en la sociedad, advierten sobre la importancia de que las universidades presten especial atención y den cabal seguimiento a las trayectorias de los alumnos para que culminen de la mejor forma posible sus estudios universitarios. Sin embargo, la falta de tiempo, dicho más claramente, la dificultad para administrar mejor el tiempo, es el factor principal por el que los egresados posponen, retrasan o se les dificulta más esta etapa final de sus estudios.

Es preciso que se analicen con sumo cuidado las modalidades de titulación, se piense en la vinculación universitaria con la sociedad, en particular con el mercado laboral, no se descuide el trabajo de tutorías, se preste atención a la oferta de seminarios de titulación, a la calidad del programa de estudios y a todo el proceso educativo desde el ingreso hasta el proceso mismo de titulación con el objeto de lograr un índice de eficiencia terminal institucional equilibrada para la universidad. Pues cabe preguntarse ¿qué reflejan aquellas universidades con mayores índices de titulación?, ¿Qué están haciendo unas y otras no si en casi todas las modalidades de titulación son las mismas? Se debe considerar la opinión de los mismos alumnos.

\section{Bibliografía}

ANUIES (1988). Esquema básico para estudios de egresados en Educación Superior. México, Asociación Nacional de Universidades e Instituciones de Educación Superior, ANUIES.

ANUIES (2001). Deserción, rezago y eficiencia terminal en las IES. Propuesta metodológica para su estudio, México, Asociación Nacional de Universidades e Instituciones de Educación Superior, ANUIES.

Anzaldo, M., Morales, E. y Nolasco, S. (2001). Vida académica y eficiencia terminal del nivel superior de la Universidad Autónoma de Nayarit. México, Universidad Autónoma de Nayarit.

Becker, G. (1983). El capital humano, Madrid, Alianza editorial.

Camarena, R. (2007). Reflexiones en torno al rendimiento escolar y a la eficiencia terminal. En Revista de la Educación Superior, México, Enero- marzo, Vol.14, No. 1, pp. 34-63. 
Destinoble, A. G. (2006), El capital humano en las teorías del crecimiento económico. Chihuahua. Universidad Autónoma de Chihuahua- Escuela de Economía Internacional.

Edel, R., Duarte, V., y Hernández, S. (2006). La eficiencia terminal en la educación superior privada en México: estudio de caso de la Universidad Cristóbal Colón. En Revista de la Universidad Cristóbal Colón Número 19, edición digital a texto completo en www.eumed.net/ rev/rucc/19/

Frame, F. (2004). Eficiencia terminal de las instituciones mexicanas de educación superior. México, ANUIES.

Legorreta, Y. (2001). Deserción, rezago y eficiencia terminal en las IES: Factores normativos que obstaculizan el egreso y la titulación, México, Asociación Nacional de Universidades e Instituciones de Educación Superior, ANUIES.

López, N. Salvo, B. y García, G. (1989). Consideraciones en torno a la titulación en las instituciones de educación superior. Revista de la Educación Superior, Vol. 18, No. 1 (69) enero-marzo 1989.

Lugo, A. (2003). Los escenarios de la educación superior. Disponible en: http://www.sappiens. com/CASTELLANO/articulos.nsf/Educadores/Los_escenarios_de_la_Educación_Superior/2A 475191E0EC4AB4C1256E050014389E!opendocument

Norting, J. (2007). Ponencia sobre capital humano, la experiencia de Suecia. En: http://www. swedenabroad.com/page_67773.aspx.

Piore, M. (1983). Unemployment and Inflation: Institutionalist and Structuralist Views. USA, Sharpe Press.

Rodríguez, C. (2006). Políticas de formación de los RRHH frente a la globalización - ¿Qué escenario es el óptimo? En: http://www.gestiopolis.com/recursos/documentos/fulldocs/rrhh/ rrhhglobalizacion.htm

Romo, A. (2003). De la eficiencia terminal en la calidad de la educación superior. Revista Innovación Educativa, Julio-agosto. No. 15, pp. 4-15.

Valenti, G. (2006). Las pistas del seguimiento: el puente necesario entre educación y mercado laboral. México, Facultad Latinoamericana de Ciencias Sociales. FLACSO.

\section{Notas}

${ }^{1}$ Camarena (2007) conceptualiza la eficiencia terminal como un indicador cuantitativo de los logros obtenidos por un establecimiento escolar, y se le utiliza como pauta de evaluación del funcionamiento y rendimiento de las propias escuelas. Anzaldo, Morales y Nolasco (2001) la definen como el proceso educativo que se desarrolla durante un determinado número de ciclos preestablecidos hasta la conclusión de un plan de estudios. Mencionan que este concepto no siempre se presenta aislado ya que es el resultado de un proceso educativo y forma parte de una serie de acontecimientos tanto académicos como sociales, internos y externos de la institución educativa y por medio del cual se identifican indicadores institucionales los cuales se centran en el desempeño escolar de los estudiantes y de la institución misma. 